\title{
Concepts of Regional Therapies for Advanced Malignancy
}

\author{
T. Clark Gamblin, $\mathrm{MD}^{1}$, H. Richard Alexander, $\mathrm{MD}^{2}$, Robert Edwards, $\mathrm{MD}^{3}$, and David L. Bartlett, $\mathrm{MD}^{4}$ \\ ${ }^{1}$ Division of Surgical Oncology, Department of Surgery, Medical College of Wisconsin, Milwaukee, WI; ${ }^{2}$ Department \\ of Surgery, University of Maryland, Baltimore, MD; ${ }^{3}$ Division of Gynecologic Oncology, University of Pittsburgh, \\ Pittsburgh, PA; ${ }^{4}$ Division of Surgical Oncology, Department of Surgery, University of Pittsburgh, Pittsburgh, PA
}

Management approaches for regionally advanced cancers include cytoreductive surgery and hyperthermic intraperitoneal chemotherapy (HIPEC), isolated limb infusion and perfusion, isolated liver perfusion, hepatic artery directed therapies, and other novel therapies, such as thoracic and pelvic perfusion. Surgical oncologists are incorporating these approaches with increasing frequency worldwide as safety, morbidity rate, and therapeutic considerations are better understood. ${ }^{1}$ These advances in regional treatment options have challenged the staging systems, examined the quality of life associated with treatment, and sought to improve patient survival for diseases with limited options. Recent improvements in the understanding of tumor biology have allowed for improved selection, and in some cases a shift from palliative to a curative intent.

In this issue of Annals of Surgical Oncology, a series of articles from the Sixth International Symposium on Regional Cancer Therapies have been solicited to offer an update on the management of advanced malignancies. These articles capture and highlight the advances that are being made in the surgical delivery of regional therapy such as isolated limb infusion in contrast to a perfusion, the improved staging, and better understanding of histology and improvement of technique and outcomes for patients with carcinomatosis.

In the first article, quality of life after isolated limb infusion for in-transit melanoma and merkel cell carcinoma is shown to have common postprocedure symptoms of skin changes, edema, and numbness; however, no patient in the series showed residual functional impairment of the treated limb. ${ }^{2}$ This work provides important insight into long-term toxic effects for isolated limb therapy. ${ }^{3}$

(C) Society of Surgical Oncology 2012

Published Online: 3 February 2012

T. C. Gamblin, MD

e-mail: tcgamblin@mcw.edu
In the second article, staging of histological subtypes of appendiceal tumors is examined utilizing the SEER data set. ${ }^{4}$ Patients were classified into subsets of adenocarcinoma, mucinous adenocarcinoma, signet ring cell type, goblet cell carcinoid, and malignant carcinoid. The proposed model highlights the significant differences between same stage histologies and provides a simple clinical tool to prognosticate the disease process.

Next, a large series of patients with mucinous appendiceal peritoneal carcinomatosis was assessed to examine postoperative and oncologic outcomes after cytoreductive surgery and HIPEC. ${ }^{5}$ This article demonstrates adequate cytoreduction in $82 \%$ and major morbidity in $25 \%$ of cases. ${ }^{6}$ Tumor grade, cytoreduction achieved, previous chemotherapy, and preoperative peritoneal carcinomatosis index (PCI) score are reported to be significant predictors of time to recurrence.

The challenges of assessing the extent of carcinomatosis is addressed in the fourth article where authors examined diffusion-weighted and gadolinium enhanced MR imaging in an attempt to more accurately predict the PCI score. ${ }^{7,8}$ They found the MRI PCI and surgical assessment PCI to have no significant difference. This radiation sparing modality holds potential for noninvasive screening and selection of patients for HIPEC and may allow for more thoughtful operative selection.

Variations in the tumor biology of mucinous appendiceal cancers and the expression of inflammatory mediators led authors in the next article to consider the role of antiinflammatory agents to target inhibition of mucin in appendiceal neoplasms with pseudomyxoma peritonei (PMP). ${ }^{9}$ This work is important in a murine xenograft model, because it shows a potential role for targeted therapy to reduce mucin production by examining secretory mucins (MUC2) and the potentials of dexamethasone and Celebrex. ${ }^{10}$ 
The final two articles are clinical outcome reports focused on cytoreductive surgery and HIPEC for advanced abdominal malignancies. ${ }^{11,12}$ One article specifically examines the subset of peritoneal sarcomatosis. ${ }^{13}$ In this paper, authors point to the ability to perform complete cytoreduction and a low PCI score as important predictors in patient selection. This manuscript provides a thoughtful application of HIPEC for sarcoma and a helpful review of the pathology specific literature. The last article utilizes two case-matched groups with diffuse peritoneal mesothelioma. They report routine complete parietal peritonectomy compared with selective parietal peritonectomy. ${ }^{14}$ In their study, those who underwent complete peritonectomy had no increase in operative complications and a significantly longer overall survival. The concept of systematic complete parietal peritonectomy is new and holds potential to impact surgical decision-making.

In summary, it is our hope that this series of articles provides an important educational resource regarding some of the most challenging clinical situations of advanced malignancy. These articles are a small representation of the novel surgical therapies occurring for patients with advanced regionally confined malignancy. Ongoing performance and presentation of basic and clinical research in the field of regional therapy will improve the outlook for these unfortunate patients.

\section{REFERENCES}

1. Esquivel J, Sticca R, Sugarbaker P, et al. Cytoreductive surgery and hyperthermic intraperitoneal chemotherapy in the management of peritoneal surface malignancies of colonic origin: a consensus statement. Society of Surgical Oncology. Ann Surg Oncol. 2007;14:128-33.

2. McClaine RJ, Giglia JS, Ahmad SA, et al. Quality of life outcomes after isolated limb infusion. Ann Surg Oncol. 2012. doi: 10.1245/s10434-012-2239-0.
3. Beasley GM, Caudle A, Petersen RP, et al. A multiinstitutional experience of isolated limb infusion: defining response and toxicity in the US. J Am Coll Surg. 2009;208(5):706-15.

4. Turaga KK, Pappas SG, Gamblin TC. Importance of histologic subtype in the staging of appendiceal tumors. Ann Surg Oncol. 2012. doi:10.1245/s10434-012-2238-1.

5. Winder T, Lenz HJ. Mucinous adenocarcinomas with intraabdominal dissemination: a review of current therapy. Oncologist. 2010;15(8):836-44.

6. Salti GI, Ailabouni L, Undevia S. Cytoreductive surgery and hyperthermic intraperitoneal chemotherapy for the treatment of peritoneal sarcomatosis. Ann Surg Oncol. 2012. doi:10.1245/ s10434-012-2240-7.

7. Low RN, Barone RM. Combined diffusion-weighted and gadolinium-enhanced MRI can accurately predict the peritoneal cancer index preoperatively in patients being considered for cytoreductive surgical procedures. Ann Surg Oncol. 2012. doi: 10.1245/s10434-012-2236-3.

8. Koh JL, Tan TD, Glenn D, Morris DL. Evaluation of preoperative computed tomography in estimating peritoneal cancer index in colorectal peritoneal carcinomatosis. Ann Surg Oncol. 2009;16: 327-33.

9. Choudry HA, Mavanur A, O'Malley ME, et al. Chronic antiinflammatory drug therapy inhibits gel-forming mucin production in a murine xenograft model of human pseudomyxoma peritonei. Ann Surg Oncol. 2012. doi:10.1245/s10434-012-2242-5.

10. Mavanur AA, Parimi V, O’Malley M, Nikiforova M, Bartlett DL, Davison JM. Establishment and characterization of a murine xenograft model of appendiceal mucinous adenocarcinoma. Int $J$ Exp Pathol. 2010;91(4):357-67.

11. Austin F, Mavanur A, Sathaiah M, et al. Aggressive management of peritoneal carcinomatosis from mucinous appendiceal neoplasms. Ann Surg Oncol. 2012. doi:10.1245/s10434-012-2241-6.

12. Baratti D, Kusamura S, Cabras AD, et al. Cytoreductive surgery with selective versus complete parietal peritonectomy followed by hyperthermic intraperitoneal chemotherapy in patients with diffuse malignant peritoneal mesothelioma: A controlled study. Ann Surg Oncol. 2012. doi:10.1245/s10434-012-2237-2.

13. Rossi CR, Casali P, Kusamura S, Baratti D, Deraco M. The consensus statement on the locoregional treatment of abdominal sarcomatosis. J Surg Oncol. 2008;98(4):291-4.

14. Sugarbaker PH. Peritonectomy procedures. Ann Surg. 1995;221: $29-42$. 